\title{
Modelling and Simulation Tool for the Analysis of Electric Power Quality Issues
}

\author{
F. Magnago ${ }^{1}$, C. Reineri ${ }^{1}$, S. Lovera ${ }^{1}$, R. Lima $^{1}$, and E. Belenguer ${ }^{2}$ \\ ${ }^{1}$ Department of Electrical Engineering \\ Universidad Nacional de Río Cuarto \\ Ruta 36 km 601, 5800 Río Cuarto (Argentina) \\ phone:+54 358 676246, fax:+ 54358 676246, e-mail: fmagnago@ing.unrc.edu.ar,creineri@ing.unrc.edu.ar \\ ${ }^{2}$ Department of Technology \\ Universitat Jaume I \\ Campus del Riu Sec. Avda. de Vincent Sos Baynat s/n 12071, Castelló de la Plana (Spain)
}

\begin{abstract}
In this paper a software tool developed for analysing the power quality of electric systems is described. The program provides accurate modelling of non-linear elements that can be included into typical electric network. Users may choose to build the network or select between typical distribution networks provided within the program. The software allows the user to simulate an entire electric network or input real time signals in order to characterize, classify, and analyse power quality events. It provides a fast and low cost solution for power quality analysis. An example is included in the paper to illustrate the usage and capabilities of the program.
\end{abstract}

\section{Key words}

Power quality, distribution systems

\section{Introduction}

Electric power system elements dramatically changed from prominent linear to highly non linear. These non linear elements are due to the increased use of elements such as arc furnaces, FACTS devices, dc converters, uninterruptible power supply (UPS), or variable speed motor drives. These non linear elements considerably affect the quality of the electric power supply. On the other hand, these elements are sensitive to the variation of power quality parameters such as harmonics, sags, under and over voltages, flickers, etc. Therefore, power engineers increased the attention on electric power quality topics. As a consequence, it increments also the research activities on the topic.

A wide range of possible research directions have been identified in the general area of power quality. Among them, an important portion of the power quality research is devoted to issues such as [1]:

1) modelling of non linear loads,

2) time domain simulation, and

3) steady state solutions for electrical systems.
These topics make imperative the development of new software tools to assist these matters.

When analysing power quality events, the first step is to look at the disturbances and to whom they affect. The disturbances may be generated at the utility side affecting the customers, or may be generated by customers causing disturbances that affect the utility side. Hence, an important task is to develop strategies for assessment of customers power quality problems, studying them in order to find the best solution.

In order to asses the quality of the power, different tools have been developed. Halpin [2] describes simulation methods for analysing and evaluating some mitigation of flicker problem, Abur [1] proposes a testing laboratory for power quality studies, Liao [3] provides a new software implementation for detection and classification.

The tasks of detecting and classifying of power quality events, characterizing, locating, and analysing events, are closely related.

Analyses in house reduce costs and speed up the implementation of a solution. Therefore, it is important to carry out the study of these topics utilizing the same software environment.

This paper describes a new software tool that allows to study a wide range of power quality topics using the same software environment.

\section{Software structure}

Power quality involves monitoring and simulation of power quality phenomena on distribution systems. The integration of data collection and data management tools can quickly characterize and analyse power quality data.

Moreover, analysis tools integrated with the data base can provide a fast answer to many power quality 
problems. Based on these premises, the software was designed. The modular design allows future expansion and customisation, including a friendly user interface which novice and advance users can take advantage of it. The software allows seeing results graphically, otherwise, these results can be saved for post processing. The program also provides software applications for post processing simulation results. These applications are a signal classification tool that allows the user to identify the kind of perturbation generated by the non - linear elements (i.e. sag, flicker, etc.), a characterization tool that calculates electric power quality indexes of the signals (i.e. THD, power factor, etc.), these indexes are compared to limits recommended by international norms, and an analysis tool that provides additional post processing evaluation.

Fig. (1) shows the overall software structure. The program has been implemented using MATLAB, and has seven main modules. All these modules can be access through a friendly graphical user interface. The core of the program is the data base manager since all the applications interact among them through the information provided by the data base. Several application studies related to power quality problems can be carried out using one of the modules or a combination of them.

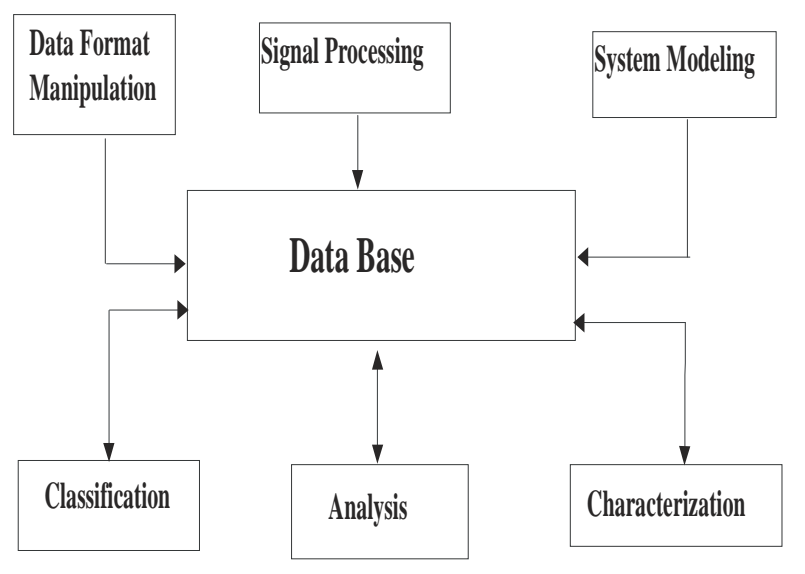

Fig. 1. Software structure

A synthetic description of each module is given next.

\section{A. System Modelling}

In order to evaluate various power quality events, such as the influence of multiple harmonic sources on a specific substation, a large sector of the electric system needs to be included. Therefore, three phase models of different power systems elements have been considered. These models are included into different libraries such as:

1) Loads: In addition to conventional shunt elements, several models that have astrong influence on power quality events are included: inverters, converters, current injections with different number of harmonics, and machines.
2) Power systems devices: To model the electric network, all necessary elements are added, for example, three phase lines, transformers, generators, and parallel devices. In order to compare with other software, small test systems have been included into the library.

3) Filters: To study how to mitigate the effect of different power quality events, active and passive filters are included. The active filters included at this moment are based on the sliding mode control strategy.

4) Measurement elements: The effect of voltage and current transducers affect the response of the electric signals. In general they operate as a filter, consequently, they need to be modelled and included into the system.

\section{B. Input Data Manipulation}

The program allows the user to generate data by modelling the system and record different quantities at any location. Another alternative is to input data from the field through a file. These data may have different formats, therefore this module provides different tools to convert data from the most popular formats to a common data format. Data can be input in ASCII, MATLAB, comtrade, or EMTP/ATP output formats.

\section{Signal Processing}

Once the data is converted into a common format and saved into the data base, the signal processing module allows to calculate frequency and phasors from the input signal, to be used as a base for indexes calculation. There are many digital algorithms applied to calculate frequency and phasors, for example, the Kalman filter, the Prony method, the discrete Fourier transform and the discrete wavelet transform. The discrete Fourier transform and the wavelet transform are the ones implemented within this module. This tool not only allows to convert the signal for future processing or analysis, but also to compare different signal processing tools. Implementing them on different types of input signals, allowing the selection of the most appropriate for each case. is converted into a common format and saved into the data base, the signal processing module allows to calculate frequency and phasors from the input signal, to be used as a base for indexes calculation. There are many digital algorithms applied to calculate frequency and phasors, for example, the Kalman filter, the Prony method, the discrete Fourier transform and the discrete wavelet transform. The discrete Fourier transform and the wavelet transform are the ones implemented within this module. This tool not only allows to convert the signal for future processing or analysis, but also to compare different signal processing tools. Implementing them on different types of input signals, allowing the selection of the most appropriate for each case. 


\section{Classification}

It is important to identify the power quality events based on time and frequency spectrums, in order to take an appropriate corrective action. Among different types of events, the program can identify and classify them into one of the following types:
1) Voltage sag due to a fault
2) Voltage sag due to a motor starting
3) flicker
4) .harmonic distortion
5) .transient
6) .notch

\section{E. Characterization}

Once the event is classified, it is necessary to have some measure of how serious the perturbation is. This is done by calculating several types of measures called indexes [4] and comparing them with limits imposed by international standards. The program provides the calculation of the most classical measures of electric power quality events, such as:
1) Total harmonic distortion (THD)
2) Electric power
3) Power factor
4) Flicker factor
5) Unbalance factor
6) Rms values

F. Analysis

Once the characterization of a signal is done, it is important to analyse these data in order to:

1) study who is responsible for these perturbation,

2) know how the perturbation propagates through the system,

3) design corrective actions, and

4) calculate the penalizations imposed by the regulations.
For each of the above topics, different analysis should be done.

One of the implemented tools is the statistical analysis for measured data characterization. This module is explained through an example in the following section.

\section{Data Characterization}

One of the analyses implemented is the data characterization of measurement containing time varying harmonics [5]. The importance on an analysis of this type of signals has increased over the past years. Electric utilities assess the power quality of signals that contain harmonic frequencies using measurement programs. However, most of the existing standards on harmonic limits are fixed values. Considering that measurements contain time variant signals, a different analysis should be implemented.

Fig. (2) shows a block diagram of the implemented software structure. The signal is transformed to frequency domain using the Discrete Fast Fourier transform, the harmonic levels of the non steady state distorted signals are computed and different analyses to describe the data in statistical terms are made.

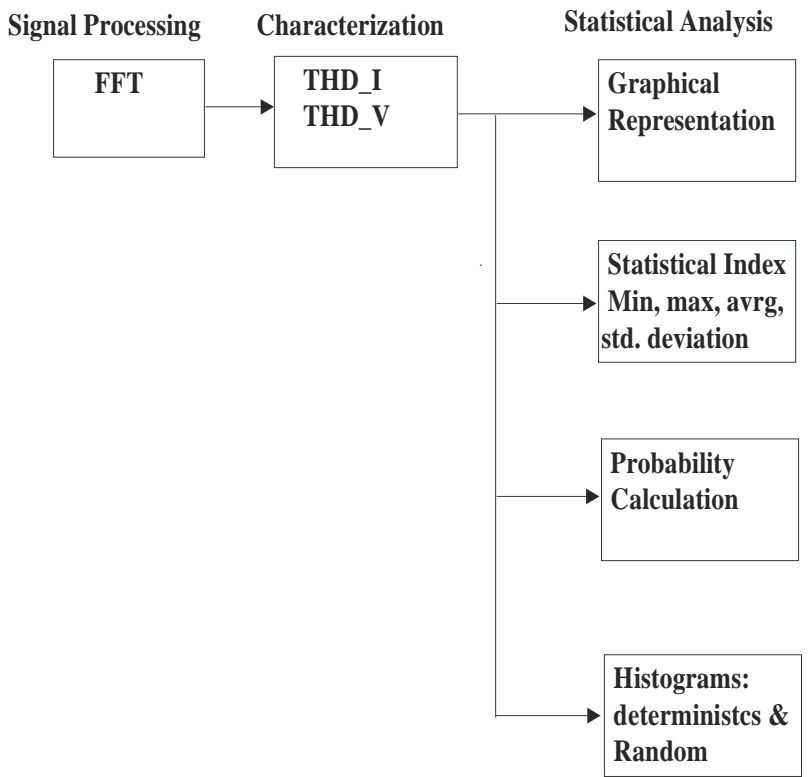

Fig. 2. Statistical analysis module

To show the features of this module, a recorded data from a bus containing a rolling mill is used as a test signal. The voltage level at the bus is $13.8 \mathrm{kV}$. The sampling rate is one sample every minute, for a period of six hours. The mill works the first three hours. Fig. (3), (4), and (5) are screen captures of the output results of the program. 
The first step is the total harmonic distortion (THD) for both the voltage and the current signals. The voltage THD in percent is shown in the left diagram of Fig. (3). Once the THDs are calculated, statistical measures are calculated for these parameters. These measures include minimum value, maximum value, mean value and standard deviation of the data set. These measures are shown on the right part of the Fig. (3).

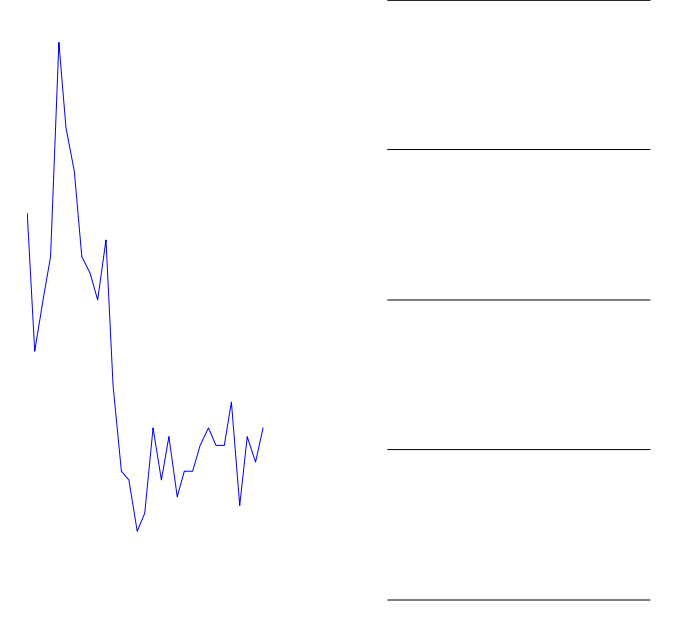

Fig. 3. Total harmonic distortion

Considering that in this example the THD data follows a Gaussian distribution, the probability density function is calculated and shown as a histogram, and is illustrate on Fig. (4).
Although these histograms provide more information than the statistical measures, they still do not provide information on when the events took place. Therefore, the probability distribution function is calculated and shown in Fig. (4) as probability of occurrence.

Finally a combined description of statistical and deterministic analysis is done and compared, shown in Fig. (5).

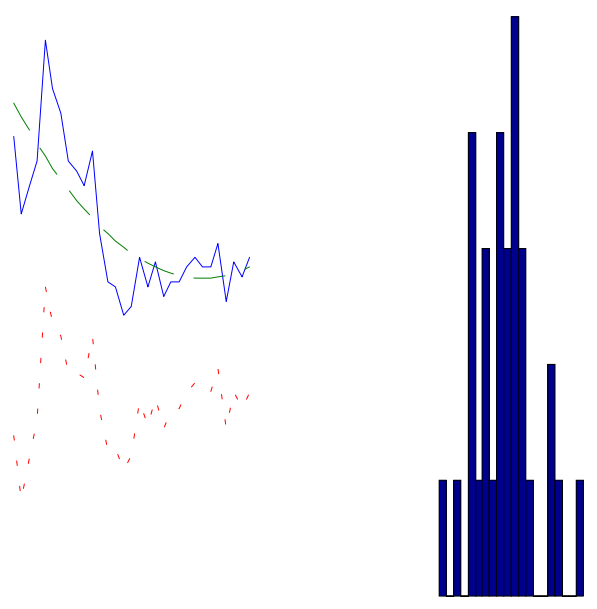

Fig. 5. Deterministic analysis module

This data characterization methods is shown as an example of the analysis feature of the program. This particular example shows potential problems associated with harmonic measurement of time varying waveforms and illustrate how through the modelling and simulation the topic can be study.

\section{Conclusion}

A new software tool that allows to study a wide range of power quality topics using the same software environment was described. The software designed in a modular fashion is capable to simulate an entire electric network, input real time signals, characterize, classify, and analyse power quality events. Electrical systems can be modelled, simulated, and studied under the presence of non linear loads using this software, providing a fast and reliable answer to several of the electric power quality issues.

Fig. 4. Statistical distribution 


\section{References}

[1] A. Abur, M. Kezunovic, "A Simulation and Testing Laboratory for Addressing Power Quality Issues in Power Systems", IEEE Transactions on Power Systems, Vol. 14, no. 1, pp. 3-8, February 1999.

[2] S. Halpin, R. Burch, "An Improved Simulation Approach for the Analysis of Voltage Flicker and the Evaluation of Mitigation Strategies in Power Systems", 1996 IEEE/PES Summer Meeting, July 28, 1996, Denver, Colorado.

[3] M. Kezunovic, Y. Liao, "A Novel Software Implementation Concept for Power Quality Study", IEEE Transactions on Power Delivery, Vol. 17, no. 2, pp. 544-48, April 2002.

[4] G. T. Heydt, and A. W. Galli, "Transient Power Quality Problems Analysed Using Wavelets", IEEE Transactions on Power Delivery, Vol. 12, no. 2, pp. 908-915, April 1997.

[5] IEEE Harmonics Working Group, "Time Varying Harmonics: Part I - Characterizing Measured Data", IEEE Transactions on Power Delivery, Vol. 13, no. 3, pp. 938-943, July 1998. 